\title{
BEAM-BEAM DEFLECTION AS A BEAM TUNING TOOL AT THE SLAC LINEAR COLLIDER
}

\author{
W. KOSKA
}

University of Michigan, Ann Arbor, Michigan 48109, USA

P. BAMBADE *, W. KOZANECKI and N. PHINNEY

Stanford Linear Accelerator Center, Stanford University, Stanford, California 94309, USA

\author{
S.R. WAGNER \\ University of Colorado, Boulder, Colorado 80309, USA
}

Received 30 May 1989

To achieve maximum integrated luminosity at the SLAC Linear Collider, a method of noninvasive beam tuning is required. Traditional luminosity monitors based on Bhabha scattering are inadequate because of low instantaneous counting rates. Coherent deflections of one beam by the electromagnetic field of the other are sensitive not only to the relative steering of the two bunches but also to their spot sizes. A brief description of beam-beam deflection theory forms the basis for a discussirn of this phenomenon as a tool for single-beam tuning and for luminosity optimization at the interaction point of the SLC.

\section{Introduction}

The achievement of useful luminosity at the SLAC Linear Collider (SLC) requires that the $\mathrm{e}^{+}$and $\mathrm{e}^{-}$ beams be focused to rms widths of a few microns at the interaction point (IP), and be centered on each other to a fraction of this size. Several schemes have been proposed to accomplish this utilizing the beam-beam interaction itself $[1,2]$. One of these techniques is based on measurement of the deflections induced in beam trajectories by their coherent electromagnetic interaction as they pass each other at the IP with a small but nonzero impact parameter. These beam-beam deflections have recently been observed at the SLC [3]. In this paper, we discuss how beam-beam deflections may be used for optimizing luminosity at the collider. After a brief summary of the theory of deflections, we illustrate how this phenomenon is used to steer beams into collision and to optimize beam parameters at the IP.

\section{Basic equations}

We will summarize the basic equations relating to the deflection of Gaussian, upright elliptical beams as-

\footnotetext{
* Present address: Laboratories de l'Accélérateur Linéaire, Bât. 200, Orsay, France 91405.
}

suming that effects from disruption [4] are negligible and that the transverse rms beam widths do not change appreciably during the collision. The general expression for the deflection of one beam (the probe) by an oppositely charged beam (the target) moving on an opposing trajectory is:

$$
\begin{aligned}
& \left\langle\theta_{x, y}\right\rangle=\frac{-2 r_{\mathrm{e}} N_{t} \Delta_{x, y}}{\gamma} \\
& \times \int_{0}^{\infty} \mathrm{d} t \frac{\exp \left[-\left(\frac{\Delta_{x}^{2}}{\left(t+2 \Sigma_{x}^{2}\right)}+\frac{\Delta_{y}^{2}}{\left(t+2 \Sigma_{y}^{2}\right)}\right)\right]}{\left(t+2 \Sigma_{x, y}^{2}\right)\left(t+2 \Sigma_{x}^{2}\right)^{1 / 2}\left(t+2 \Sigma_{y}^{2}\right)^{1 / 2}},
\end{aligned}
$$

where $N_{\mathrm{t}}$ is the number of particles in the target beam, $r_{\mathrm{e}}$ is the classical radius of the electron, $\gamma$ is the Lorentz factor, $\Delta_{x}\left(\Delta_{y}\right)$ is the distance between beam centers and $\Sigma_{x}^{2}=\sigma_{\mathrm{p}, x}^{2}+\sigma_{1, x}^{2}\left(\Sigma_{y}^{2}=\sigma_{\mathrm{p}, y}^{2}+\sigma_{1, y}^{2}\right)$ is the sum of the squares of the probe and target beam sizes. We will refer to $\Sigma_{x}$ and $\Sigma_{y}$ as the interaction radii in $x$ and $y$ of the beams. The integration in this expression can be performed analytically if we assume that $\Sigma_{x}=\Sigma_{y}=\Sigma$ (this includes the case of round probe and target beams). With $\Delta=\left(\Delta_{x}^{2}+\Delta_{y}^{2}\right)^{1 / 2}$ the result is:

$$
\left\langle\theta_{x, y}\right\rangle=\frac{-2 r_{\mathrm{c}} N_{\mathrm{t}} \Delta_{x, y}}{\gamma \Delta}\left(\frac{1-\exp \left(-\frac{\Delta^{2}}{2 \Sigma^{2}}\right)}{\Delta}\right) .
$$




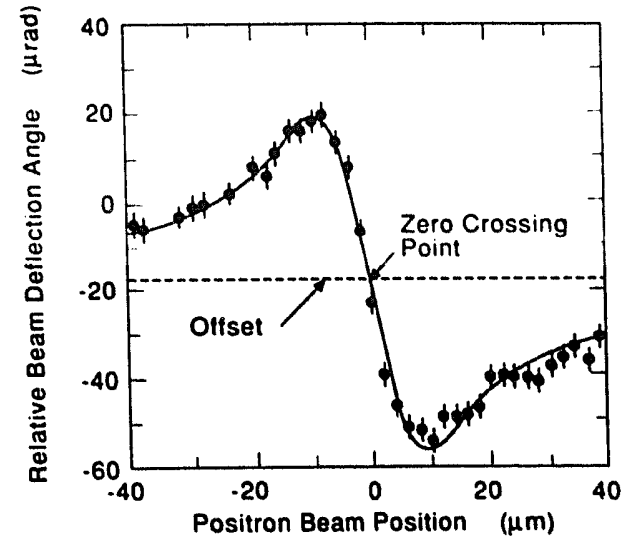

Fig. 1. A measured $\mathrm{e}^{+}$beam deflection scan in $y$ after alignment in $x$. The errors on the points are derived from the resolution of the BPMs. The fit to the data was obtained from eq. (7). Deflections are relative to an arbitrary constant offset as described in the text, and the zero-crossing point corresponds to where the beam-beam deflection curve crosses this offset.

When the beams are centered in either dimension (i.e., $\Delta_{x}=0$ or $\Delta_{y}=0$ ), the corresponding deflection angle is zero. We define this to be the zero-crossing point of the beam-beam deflection scan in that plane (fig. 1). Hence, steering the beams to produce no deflection in either dimension will maximize luminosity. Furthermore, we note that when $\Delta_{x}^{2} \ll \Sigma_{x}^{2}$ and $\Delta_{y}^{2} \ll \Sigma_{y}^{2}$. then:

$\left\langle\theta_{x, y}\right\rangle \approx S_{x, y} \Delta_{x, y}$,

where

$S_{x, y}=\left.\frac{\partial\left\langle\theta_{x, y}\right\rangle}{\partial \Delta_{x, y}}\right|_{\Delta_{x, y}=0}=\frac{2 N_{\mathrm{t}} r_{\mathrm{c}}}{\gamma} \frac{1}{\Sigma_{x, y}\left(\Sigma_{x}+\Sigma_{y}\right)}$

is the slope of the function described by eq. (1) at the zero-crossing point.

The luminosity is:

$L=\frac{N_{1} N_{2} f}{2 \pi \Sigma_{x} \Sigma_{y}}$

where $N_{1}$ and $N_{2}$ are the number of particles in beams 1 and 2 respectively, and $f$ is the repetition rate of the collider. inerefore, $L$ can be determined if $N_{1}, N_{2}, \Sigma_{x}$ and $\Sigma_{y}$ can be measured. Alternatively, $L$ can also be determined if $S_{x}$ and $S_{y}$ for beam 1 (referred to as $S_{1, x}$ and $\left.S_{1, y}\right)$ or beam $2\left(S_{2, x}\right.$ and $\left.S_{2, y}\right)$ are measured, since from eqs. (3) and (4):

$L=\frac{N_{1} f \gamma}{4 \pi r_{\mathrm{e}}}\left(S_{1, x}+S_{1, y}\right)=\frac{N_{2} f \gamma}{4 \pi r_{\mathrm{e}}}\left(S_{2, x}+S_{2, y}\right)$.

From this latter equation it can be seen that luminosity is maximum when the slopes $S_{x}$ and $S_{y}$ are maximum.

\section{Beam steering}

A typical beam-beam deflection scan is shown in fig. 1. It was obtained by scanning the positron beam vertically across the electron beam in steps of iwo microns At each step, the deflection angle was measured for three consecutive beam pulses and averaged. The entire scan was accomplished in 120 pulses. This procedure is described in detail in ref. [3]. The fit overlaying the data has the form (see eq. (2)):

$A+B\left(\frac{1-\exp \left(-\frac{\Delta^{2}}{2 \Sigma^{2}}\right)}{\Delta}\right)$

which was derived for the special case in which the beams are aligned in the out-of-plane [5] direction and the interaction radii of the beams are equal in $x$ and $y$ $\left(\Sigma_{x}=\Sigma_{y}\right)$. The constant term $A$ is used to remove an offset which results from residual misalignment or electrical offsets in the individual BPMs used to make the deflection measurement [6], and $B$ is a constant proportional to the number of particles in the target beam. Using this simple form allows us to do fast on-line fitting to the data, but it may not give the correct results when the beams are very elliptical, or grossly misaligned in the out-of-plane direction. However, as a consequence of the symmetry of the equation, the fit parameter $\Delta$, corresponding to the zero-crossing point (which indicates the beams are aligned), is still accurate. Hence,

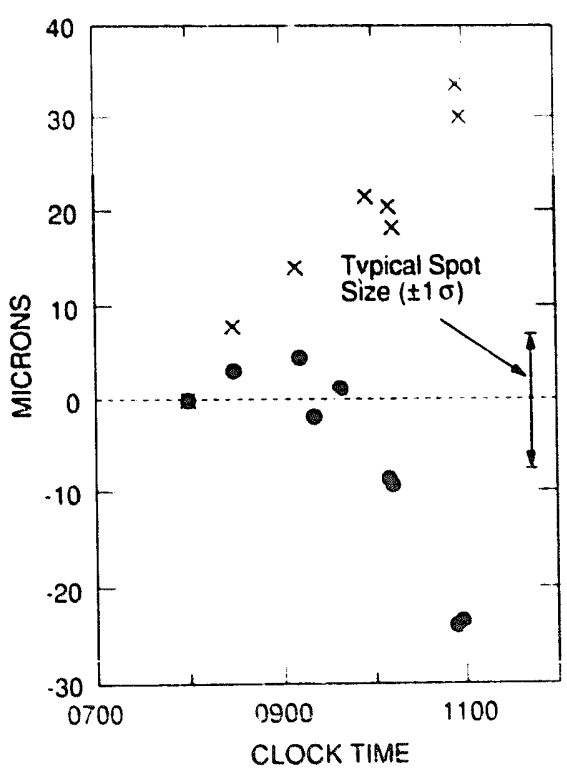

Fig. 2. The cumulative motion of the beam-beam interaction point vs time for the horizontal $(X)$ and vertical) (๑) directions. The motion is relative to the position where the beams were initially centered at $\mathbf{0 8 0 0}$. These data were obtained from deflection scans which were periodically made to recenter the beams. The beam size was typically $6-7 \mu \mathrm{m}$ during this time. 
iteration of beam-beam deflection scans in $x$ and $y$ can be used to remove misalignment in the out-of-plane direction and it has been shown using a simulation that alignment to better than $10 \%$ of $\Sigma$ can be achieved when the beams have aspect ratios $\left(\sigma_{x} / \sigma_{y}\right)$ in the range from 0.3 to 3 , assuming $N \geqq 10^{10}$. Beam alignment using the deflection technique is standard procedure at the SLC. Fig. 2 illustrates a time history of the horizontal and vertical beam-beam interaction point as determined using deflections [7].

\section{Beam tuning}

Determination of spot size at the IP is critical for several tuning and diagnostic procedures (e.g., waist positioning) $[8,9]$. At lov beam intensities, measurements of the spot sizes can be made using fine carbon fibers [10]. However, at high beam intensities $(\geq 1.0 \times$ $10^{10} \mathrm{e}^{-}$/bunch) and small spot sizes $(\leq 3 \mu \mathrm{m})$, the thermal shock experienced by the fiber when hit by the beam is expected to destroy it in a single pulse. For this reason, it is necessary to develop alternative measurement techniques based on the beam-beam interaction itself. An additional benefit of beam-beam deflection is that it is noninvasive and can be performed during cperation of the SLC for high energy physics data taking.

Opticai tuning at the IP generally involves varying some optical component and measuring the effect on the beam size. We will describe the procedure used to position the waists of the beams at the IP. The square of the interaction radius in $x$ at the IP, when the waists of beams 1 and 2 are longitudinally displaced from the IP by $\left(z_{1}-z_{1 P}\right)$ and $\left(z_{2}-z_{1 P}\right)$ respectively, can be expressed as [11]:

$$
\begin{aligned}
\Sigma_{x}^{2}= & \epsilon_{x, 1} \beta_{x, 1}^{*}+\epsilon_{x, 2} \beta_{x, 2}^{*}+\frac{\epsilon_{x, 1}}{\beta_{x, 1}^{*}}\left(z_{1}-z_{1 \mathrm{P}}\right)^{2} \\
& +\frac{\epsilon_{x, 2}}{\beta_{x, 2}^{*}}\left(z_{2}-z_{1 \mathrm{P}}\right)^{2},
\end{aligned}
$$

where $\epsilon_{x, 1}\left(\epsilon_{x, 2}\right)$ is the emittance and $\beta_{x, 1}^{*}\left(\beta_{x, 2}^{*}\right)$ is the value of the $\beta$ function at the IP of beam 1 (2). A similar expression holds for $\Sigma_{y}^{2}$. From eq. (8), it can be seen that if the waist position of one beam (e.g., $z_{2}$ ) is held fixed and the longitudinal position of the other waist $\left(z_{1}\right)$ is varied, a plot of $\Sigma_{x}^{2}$ as a function of the longitudinal waist position of $z_{1}$ will describe a parabola The coefficients of the parabola provide information on the beam parameters and waist position relative to the interaction point.

In practice, waist positioning is performed by varying the strengths of two quadrupoles in the final demagnifying telescope in such a way as to step either the vertical or horizontal waist of a specific beam through a

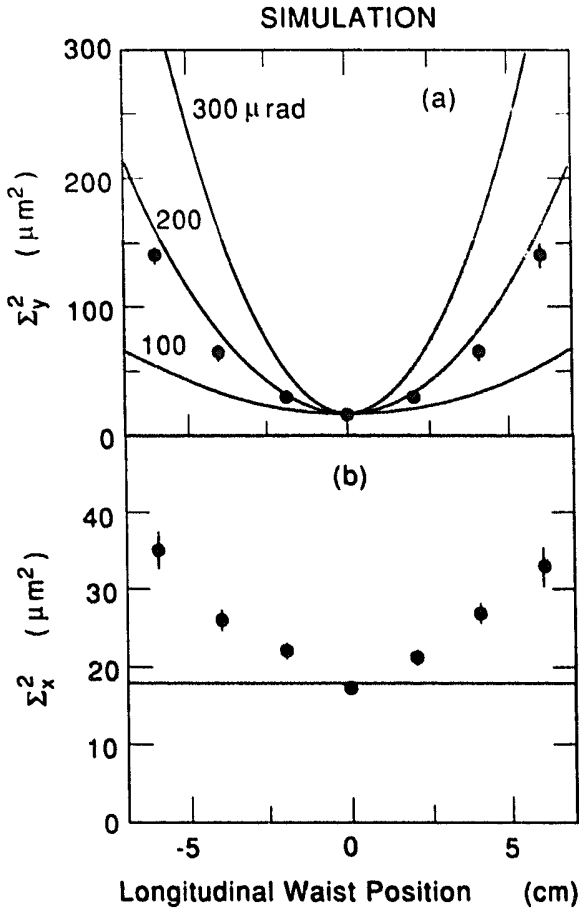

Fig. 3. The solid curves correspond to a simulation of the dependence of (a) $\Sigma_{y}^{2}$ and (b) $\Sigma_{x}^{2}$ on the longitudinal position of the electron beam vertical waist for three different beam angular divergences. The points correspond to the interaction radius as determined by the simple fitting function eq. (7) for the $200 \mu \mathrm{rad}$ case.

range specified by the operator. This is referred to as a "waist scan". After each step the beams are realigned (using the beam-beam deflection method) to compensate for quadrupole steering effects. After alignment is achieved in both planes, $\Sigma_{x}^{2}$ is obtained from the fit to the beam deflection curve. If, for example, the horizontal waist of beam 1 has been scanned, a parabolic fit to the plot of $\Sigma_{x}^{2}$ versus longitudinal waist position will yield the following three quantities: $\left[\epsilon_{x, 1} \beta_{x, 1}^{*}+\epsilon_{x, 2} \beta_{x, 2}^{*}\right.$ $\left.+\left(\epsilon_{x, 2} / \beta_{x, 2}^{*}\right)\left(z_{2}-z_{1 P}\right)^{2}\right),\left(\epsilon_{x, 1} / \beta_{x, 1}^{*}\right)$, and the setting of the quadrupoles which puts the waist at the IP. Analogous parameters are obtained when the procedure is repeated for the horizontal waist of beam 2 , and the vertical waists of both beams.

Note that during the measurement, the aspect ratio of the beam whose waist is being moved, varies. As the beam becomes less round the determination of $\Sigma_{x}^{2}$, using eq. (7), becomes systematically worse. A fit to a parabola will then yield incorrect parameter values. However, since the data are synmetric about the minimum $\Sigma_{x}^{2}$, the quadrupole settings which will position the beam waist at the IP are accurately determined. Hence, the simple, fast, on-line fitting procedure can be used to place the beam waists at the IP to optimize luminosity. Fig. 3 shows a simulation of the dependence of (a) $\Sigma_{y}^{2}$ and (b) $\Sigma_{x}^{2}$ on the longitudinal position of the 


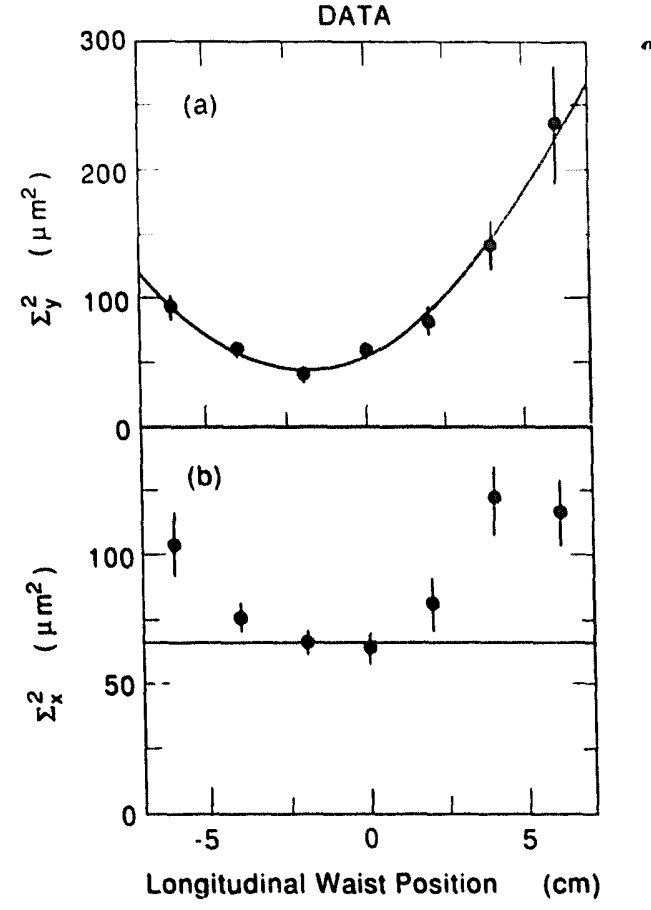

Fig. 4. A measurement of (a) $\Sigma_{y}^{2}$ and (b) $\Sigma_{x}^{2}$ vs longitudinal position of the eleciron beam vertical waist. The solid curve in the upper plot is a fit to the data using eq. (8). The solid line in the lower plot is the value of $\Sigma_{x}^{2}$ as measured with the carbon fibers.

electron beam vertical waist. The heoretical dependence is shown by the solid curves. The points correspond to the values returned by the simple fitting formula for a simulated scan using as input $200 \mu \mathrm{rad}$ for the beam angular divergence. A fit to these points indicates that the waist can be positioned at the IP to within $1 \mathrm{~mm}$, which implies a contribution to the transverse beam size due to longitudinal misalignment of less than 1\%. The fit also returns an angular divergence $\left(=\sqrt{\epsilon / \beta^{*}}\right)$ of $180 \pm 4 \mu \mathrm{rad}$, an underestimate of $10 \%$. This indicates the scale of the systematic error introduced by the simple fitting formula in the determination of beam angular divergence.

Fig. 4 shows one of the first attempts at measuring (a) $\Sigma_{y}^{2}$ and (b) $\Sigma_{x}^{2}$ as a function of longitudinal position of the electron beam vertical waist. The fit to the data yielded an angular divergence of $170 \mu \mathrm{rad}$. The minimum beam sizes were consistent with those obtained using the wire target prior to this scan. The behavior of $\Sigma_{1}^{2}$ corresponds to that which is predict $\mathrm{d}$ by the simulation.

The beam-beam deflection techniqi e has been incorporated into two other tuning proce lures. The first of these involves cross-plane coupling which had been removed by performing a scan (similar to that described for waist positioning) with skew quadrupoles, while measuring changes in the beam profile with a wire fiber.
We are currently using measurements of the interaction radius obtained with beam-beam deflections, in place of the wire measurements. The second of these procedures cancels residual spatial dispersion at the IP by minimizing the spot size using closed. dispersion-generating. trajectory bumps in the chromatic correction section of the Final Focus System as described in ref. [9]. We have again successfully replaced the wire measurements of the beam size with measurements of the interaction radius obtained with deflection scans.

\section{Future applications}

In the future it will be possible to continuously monitor beam-beam deflections in a microcomputer [12]. This will be used as part of a fast feedback program to maintain the beams in collision. The microcomputer will also perform periodic short scans around the zero-crossing point to measure the slope of the deflection curve. The slope measurement can be used to monitor luminosity through eq. (6). In addition the slope, when properly corrected for fluctuations in beam intensity, is very sensitive to variations in beam size, as illustrated in fig. 5. As shown in fig. 6. the slope is most sensitive to changes in the beam size in the direction of the scan. This provides a method for determining if one or both beams has degraded in the $x$ or $y$ dimension. The upper scale in this figure relates the machine luminosity to the slope. Monitoring the slope will provide a technique for determining if beam parameters are

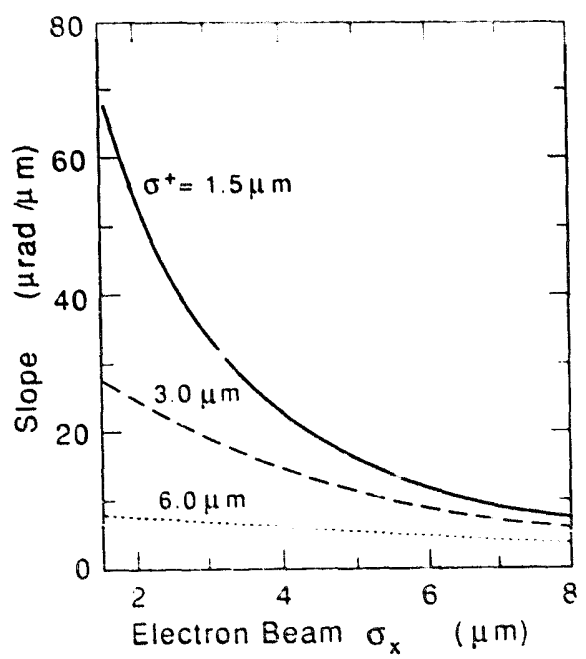

Fig 5 The calculated slope. $S_{,}$, of the def?ection curve at the zero-crossing point as a function of the $e$ beam $\sigma_{\text {, with a }}$ fixed $\sigma_{1}^{-}$of $1.5 \mu \mathrm{m}$. The curves are for round $\mathrm{e}^{+}$beams of 1.5 . 3 and $6 \mu \mathrm{m}$ in rms radius. The calculations are for beams aligned in the direction orthogonal to the scan and with intensities of $1 \times 10^{10} \mathrm{e}^{ \pm} /$bunch. 


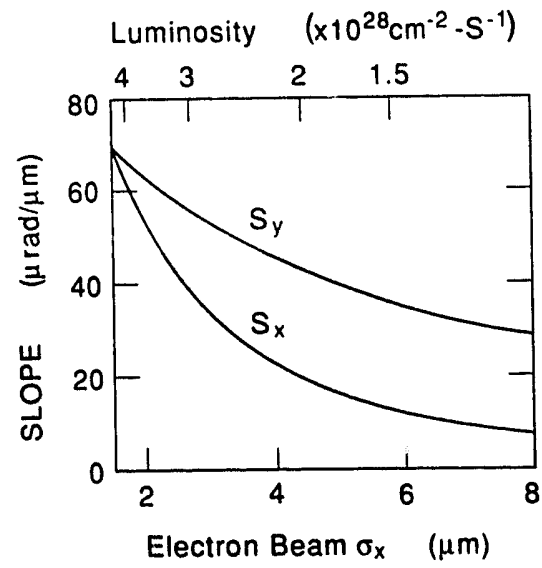

Fig. 6. The calculated slope at the zero-crossing point for horizontal $\left(S_{x}\right)$ and vertical $\left(S_{y}\right)$ scans as a function of electron beam $\sigma_{x}$. All other beam sigmas in this plot are $1.5 \mu \mathrm{m}$. The beam intensities are $1 \times 10^{10} \mathrm{e}^{ \pm} /$bunch. The upper scale indicates the luminosity under these conditions for a machine repetition rate of $120 \mathrm{~Hz}$.

changing so that corrections can be applied, possibly by an online feedback algorithm.

It should be noted that it is not possible to distinguish which beam is responsible for any measured change in the interaction radius, since this quantity is a function of the sizes of both beams. However, beamstrahlung, the radiation emitted by the individual beam particles when they are deflected, is measured simultaneously with beam-beam deflections [13]. The functional dependence of the beamstrahlung radiation on all six beam widths $\left(\sigma_{x}, \sigma_{y}\right.$ and $\sigma_{z}$ for both beams) makes it possible to extract these from data sets obtained from beam scans [14]. It is believed that beamstrahlung will eventually provide more detailed information than beam-beam deflections for diagnostic purposes and fine tuning of the beam at the IP. Combining beamstrahlung and beam-beam deflection data wili provide a powerful tool for monitoring the beams ant diagnosing changes.

\section{Conclusion}

Beam-beam deflection measurements are used to center the beams on each other to better than $10 \%$ of their interaction radii, to position the beam waists longitudinally to within $1 \mathrm{~mm}$ of the IP, and to estimate the beam angular divergence. Other, more complex tuning procedures used to correct cross-plane coupling and cancel dispersion at the IP have also been successfully adapted to use deflection measurements. Future applications include online luminosity monitoring and optical feedback.

\section{Acknowledgements}

We wish to acknowledge the many contributions to this work by the SLC staff and the Final Focus commissioning group. We would also like to thank Prof. $\mathrm{H}$. De Staebler for many useful discussions and a careful reading of this manuscript.

This work was supported in part by the Department of Energy, contracts DE-AC-2-76ER01112, DE-AC0286ER4-253, and DE-AC03-76SF00515.

\section{Reference:}

[1] P. Bambade and R. Erickson, in: Proc. Linear Accelerator Conf. (LINAC86), Stanford Linear Accelerator Center, Stanford, California (1986) p. 475.

[2] R.C. Field, Nucl. Instr. and Meth. A 265 (1988) 167.

[3] P. Bambade et al., Phys. Rev. Lett. 62 (1989) 2949.

[4] Disruption occurs when the electromagnetic interaction between beams is so intense that the particle distribution within the bunches changes during collision. This effect becomes nonnegligible for intensities greater than $3 \times 10^{10}$ when the beams are at their design RMS width of $1.5 \mu \mathrm{m}$.

[5] We refer to deffections which lie in the plane defined by the beam and scan axis directions as "in-plane", and to those orthogonal to this plane as "out-of-plane". If the beams are aligned in the out-of-plane direction, then the out-of-plane deflections are zero and the term $\Delta_{x, y} / \Delta=1$ in eq. (2) for in-plane deflections.

[6] W.A. Koska and S.R. Wagner, SLAC CN-365, Stanford Linear Accelerator Center, Stanford, California (August 1988) unpublished.

[7] D.L. Burke, presented at the Meeting of the APS Division of Particles and Fields, Storrs, Connecticut (August 1988) SLAC-PUB-4851.

[8] N. Phinney et al., Nucl. Instr. and Meth. A, to be published.

[9] P. Bambade et al., submitted to Nucl. Instr. and Meth. A.

[10] G. Bowden et al., Nucl. Instr. and Meth. A278 (1989) 664.

[11] We assume that the beams have been previously tuned to remove dispersion at the IP and that higher-order terms are negligible.

[12] F. Rouse et al., SLAC-PUB-4950, contributed to the IEEE Particle Accelerator Conf., Chicago, IL (1989).

[13] G. Bonvicini et al., Fhys. Rev. Lett. 62 (1989) z381.

[14] C. Field, E. Gero and P. Chen, private communication. 